\title{
High-Speed, Hardware Synchronized STEM EELS Spectrum-Imaging Using a Next Generation Post-Column Imaging Filter
}

\author{
P.J. Thomas, C. Trevor, E. Strohbehn, J. Wilbrink, R. D. Twesten and A. Gubbens
}

Gatan Inc., 5794 W. Las Positas Blvd., Pleasanton, CA 94588

EELS Spectrum-Imaging in the STEM delivers high spectral resolution along with excellent spatial resolution, resulting in a powerful and versatile analytical tool for investigating a wide range of materials problems. One limitation of this technique arises from the serial nature of the spatial data acquisition; each spectrum must be read out individually, which inherently places an upper limit on the achievable spectral read rate. This in turn can adversely affect the efficiency of the technique and also the total acquisition time. Hence acquiring a large EELS spectrum-image can be both time consuming and, in some cases, dose inefficient. The advent of $\mathrm{C}_{\mathrm{s}}$ probe-corrected STEM [1] has further exacerbated this limitation, since the high intensity probe demands high spectral throughput to both maximize the usefulness of the increased beam current but also to minimize sample damage.

A new high speed STEM EELS spectrum-imaging system is described here which helps alleviate this constraint by drastically increasing the spectral throughput achievable for an imaging filter. The system delivers an increase in speed by a factor of 30 over its predecessor, and 2-3 times over the fastest commercially available dedicated EELS spectrometer. Further, this high speed acquisition is fully compatible with dual EELS acquisition which allows both core-and low-losses to be acquired in a near simultaneous manner [2]. A number of key developments have enabled this:

1. A high speed camera supporting "stacked" spectroscopy. This new read mode provides higher throughput via a proprietary approach of spectrum stacking on the CCD to increase spectral read rates and duty cycle by a significant factor.

2. Hardware synchronization electronics, which enable the probe positioning to be triggered and synchronized with the camera shutter signal with sub-microsecond precision (Fig. 1a).

3. A high speed, high voltage electrostatic drift tube, which enables dual EELS switching from high- to low-loss with minimal spectrum settling overhead ( $<10 \mathrm{us})$.

A low-loss EELS spectrum-image acquired using this system is shown in Fig. 1b. The data was acquired using a GIF Quantum imaging filter, equipped with a high speed camera, electrostatic shutter and hardware synchronization support [3]. The spectral acquisition rate was $>1000$ spectra per second, with the HAADF signal acquired simultaneously (Fig. 1c). Total acquisition time for the $400 \times 400$ pixel spectrum-image was $<3$ min. including all setup overhead. Hardware synchronization ensures excellent per pixel correspondence between the beam position and spectral acquisition, as shown by a comparison between a sub-region detail of the HAADF and EELS signal (Fig. 1e). The system will be described with practical examples to illustrate the potential of this approach.

References

[1] D.A. Muller et al, Science 319 (2008) 1073.

[2] J. Scott et al, Ultramicroscopy 108 (2008) 1586.

[3] A. Gubbens et al, Ultramicroscopy (2010, in press). 
(a) (S)TEM

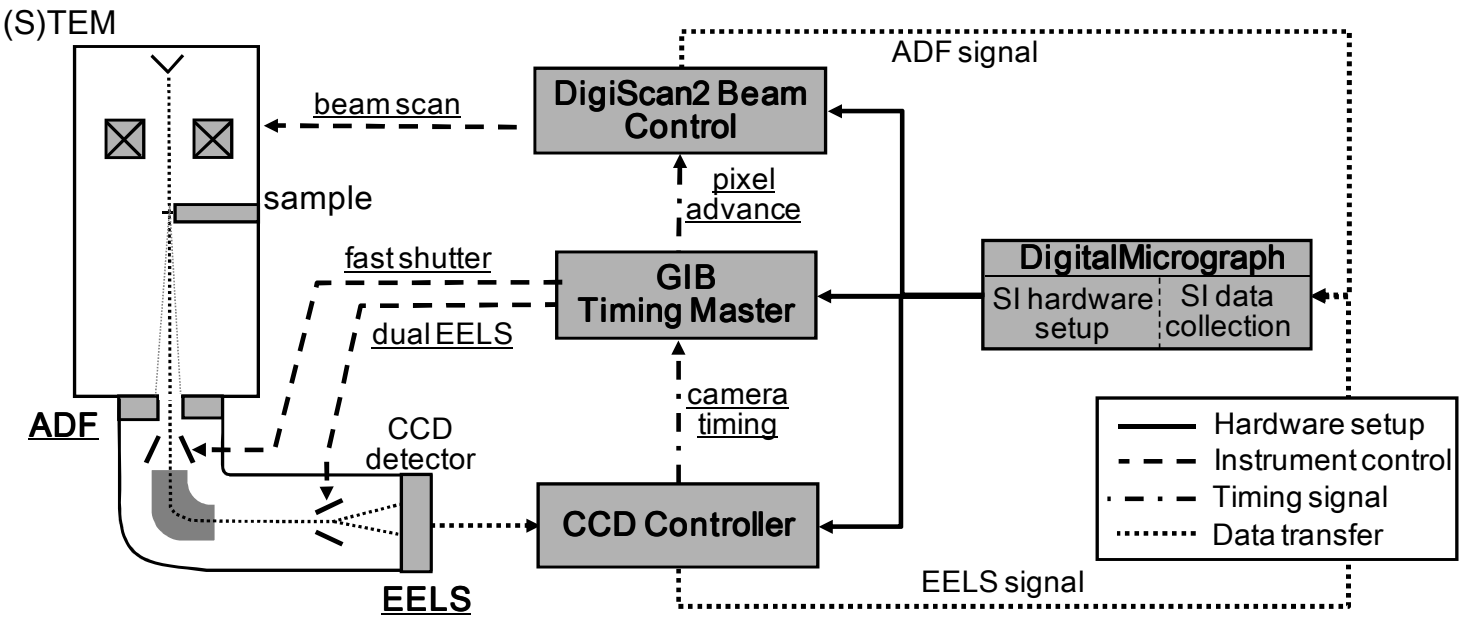

(b)

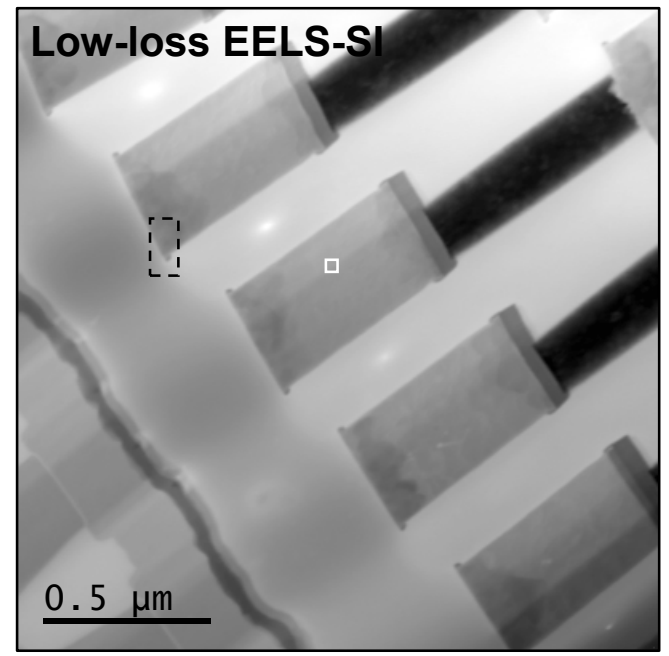

(d)

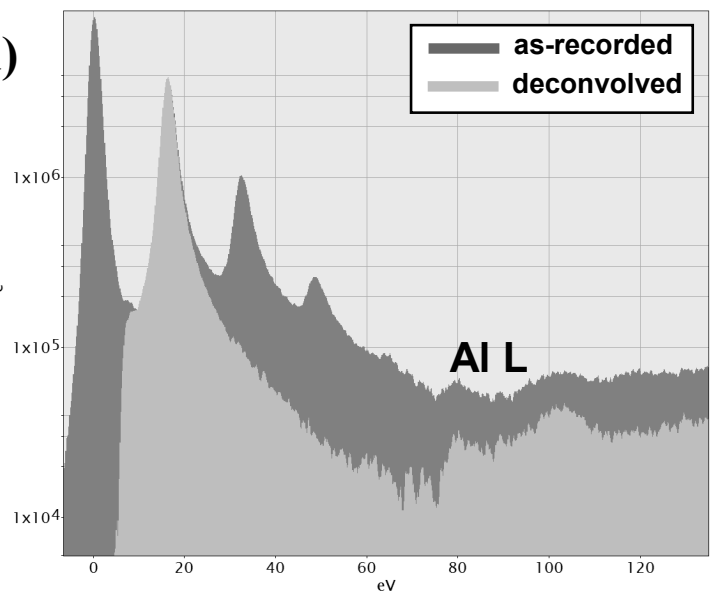

(c)

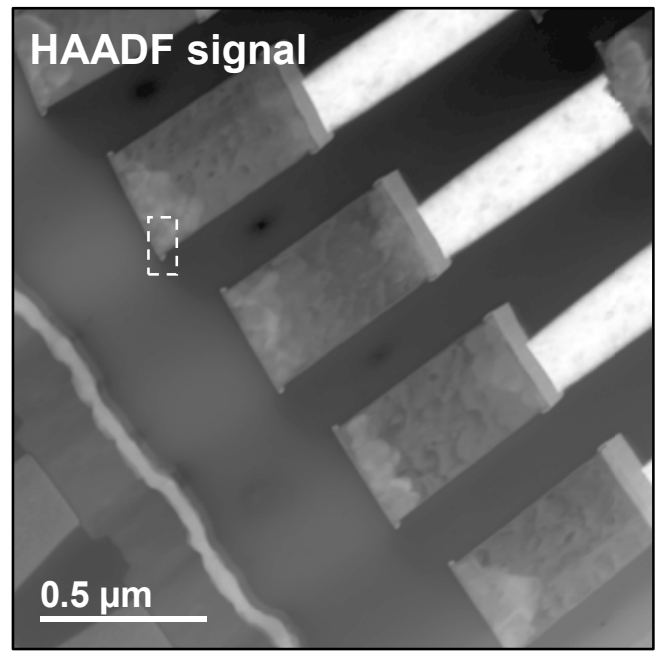

(e)

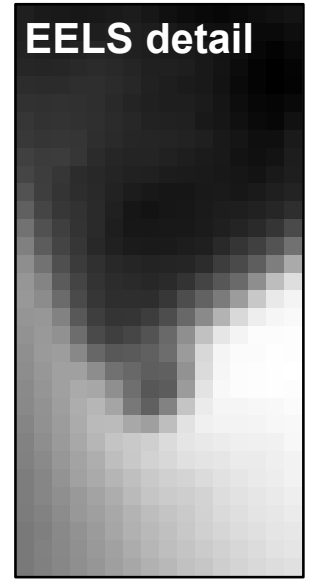

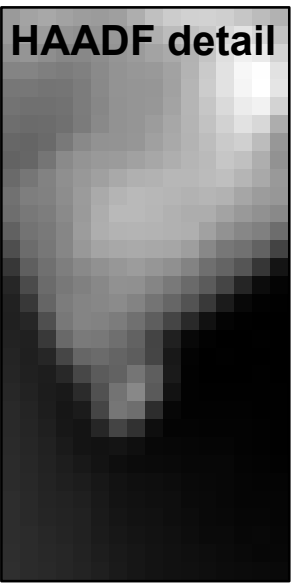

FIG. 1. (a) Schematic diagram showing the timing and data transfer configuration used in hardwaresynchronized SI. (b) Simultaneous capture of a low-loss EELS-SI (sum of the zero-loss peak shown) and (c) ADF image. Data was acquired from a commercial semi-conductor device at $>1000$ pixels/s using a GIF Quantum on Tecnai T20-LaB 6 . (d) EELS spectrum extracted and summed from the solid ROI (sum of $5 \times 5$ spectra) on the low-loss EELS-SI, before and after plural scatter deconvolution (log scale). (e) Enlarged details taken from the dashed ROIs (16x32 pixels) in Figs. b, c showing the excellent pixel-to-pixel correlation between ADF and the extracted EELS signals. 